\title{
UNA VISIÓN BIOLÓGICA PARA LA ARQUITECTURA DE INTERNET
}

\section{A BIOLOGICAL APPROACH FOR THE INTERNET ARCHITECTURE}

\section{UNE APROCHE BIOLOGIQUE POUR L'ARCHITECTURE DE L'INTERNET}

\author{
Ronildo M. da Silva \\ Instituto Federal de Bahía \\ rdasilva@ifba.edu.brl
}

(Tipo de artículo: INVESTIGACIÓN. Recibido el 02/03/2011. Aprobado el 20/05/2011)

\begin{abstract}
RESUMEN
En los últimos años ha despertado gran interés investigar acerca de la arquitectura evolutiva de Internet o del diseño de borrón y cuenta nueva para las futuras arquitecturas. Es evidente que aunque las arquitecturas de la redes se diseñan desde cero, tienden a evolucionar a medida que cambia su entorno. Esto ha suscitado algunas preguntas clave: ¿Qué hace que una arquitectura de red evolucione? ¿Qué determina la capacidad de una arquitectura de red para evolucionar a medida que cambia su entorno? En este trabajo, se revisan algunas ideas relevantes acerca de la capacidad evolutiva en la biología, y se compara con la evolución de las arquitecturas de redes computacionales. Se examina el papel de la robustez y la modularidad con respecto a la capacidad evolutiva de las redes. También se discuten los núcleos evolutivos y el equilibrio puntuado, dos conceptos importantes que pueden ser relevantes para la llamada osificación de los protocolos básicos de Internet. Finalmente, se examina la optimización, un objetivo de diseño que a menudo es de interés primordial en la ingeniería pero que no parece serlo en la biología.
\end{abstract}

\section{Palabras clave}

Arquitectura de internet, capacidad evolutiva, robustez, equilibrio puntuado.

\begin{abstract}
In the last years a great interest has emerged for the research on the evolutionary Internet architecture or the clean-slate design for the future architectures. It is evident that although the network architectures are designed from scratch, they tend to evolve as their environment changes. This has raised some key questions: What makes network architecture to evolve? What determines the ability of a network architecture to evolve while its environment changes? In this work, some relevant ideas about the evolutionary ability of biology are reviewed, and they are compared with the evolution of computer network architectures. The role of robustness and modularity with respect to the evolutionary abilities of networks is evaluated. The evolutionary core and punctuated equilibrium are also discussed; they are two important concepts that may be relevant to the so-called ossification of the basic Internet protocols. Finally, an analysis is made on the optimization, a design objective that is often of primary interest in engineering but does not seem to be in biology.
\end{abstract}

\section{Keywords}

Internet architecture, evolutionary ability, roughness, punctuated equilibrium

\section{RÉSUMÉ}

Pendant les derniers ans il a apparu un grand intérêt de réaliser des recherches sur l'architecture évolutive de l'Internet ou sur la conception de table rase pour les futures architectures. C'est évident bien que les architectures de réseaux sont conçues de zéro, elles ont la tendance à évoluer à mesure que son environnement change. $A$ partir de ça surgissent des questions clés: ¿Quelle est la cause pour l'évolution d'une architecture de réseau? ¿Qu'est-ce qui détermine la capacité d'une architecture pour évoluer à mesure que son environnement change? Dans ce travail, on révise quelques idées éminents sur la capacité évolutive dans la biologie, et sont comparés avec l'évolution des architectures des réseaux informatiques. On examine le rôle de robustesse et la modularité par rapport à la capacité évolutive des réseaux. On discute aussi les noyaux évolutifs et l'équilibre ponctué, deux concepts importants que peuvent être importants pour l'ossification des protocoles de basse de l'Internet. Finalement, on examine l'optimisation, un objectif de conception que est souvent d'intérêt primordial dans le génie mais ne le semble pas pour la biologie.

\section{Mots-clés}

Architecture de l'Internet, capacité évolutive, robustesse, équilibre ponctué. 


\section{INTRODUCCIÓN}

En los últimos 40 años Internet ha ido evolucionando de forma auto-organizada, sin un control centralizado: desde los estudios iniciales de la conmutación de paquetes en los años 60, el desarrollo de TCP/IP y LAN Ethernet en los años 70, la introducción del control de congestión DNS y TCP en los años 80, la adopción de BGP y CIDR a principios de los 90's y de los NATs a principios del 2000, hasta la introducción de nuevas tecnologías de acceso en la última década -banda ancha, LAN inalámbrica, WiMAX, etc.-, y por supuesto la continua expansión de los protocolos y servicios de la capa de aplicaciones -peer-to-peer, VoIP, vídeo streaming, juegos multi-jugador, redes sociales en línea, etc.

Recientemente, la evolución de la arquitectura de Internet se ha convertido en un importante tema de investigación por dos razones: 1) existe la preocupación de que la actual arquitectura de Internet se ha osificado, es decir, que no evoluciona tan rápido como antes, sobre todo en los protocolos básicos capas de red y transporte. Algunas extensiones arquitecturales del actual Internet, tales como IP multicast, IPv6, QoS o los nuevos protocolos de transporte como el XCP, no se han adoptado en la práctica, tal vez como resultado de dicha osificación. 2) Existe un significativo interés por diseñar arquitecturas de borrón y cuenta nueva para el futuro Internet, sin la necesidad de hacerlas compatibles con la que actualmente se tiene. Un objetivo fundamental en estos ejercicios de diseño es la capacidad evolutiva, es decir, asegurarse que una arquitectura tendrá la capacidad de evolucionar en los nuevos entornos y requisitos sin que se osifique unos años más tarde.

La capacidad evolutiva es un concepto importante en la biología [1], que explica cómo la vida se las arregla para generar drásticamente nuevos fenotipos y comportamientos complejos desde variaciones genéticas al azar, en escalas de tiempo relativamente cortas. Los biólogos han desarrollado un rico dominio de conocimiento experimental y teórico para entender la capacidad evolutiva e identificar los principios de diseño que hacen evolutivos a los organismos vivos. Estos principios incluyen la robustez y la modularidad, dos objetivos generales de diseño que están estrechamente relacionados y que tienen profundas implicaciones para la capacidad evolutiva de cualquier sistema.

En este trabajo se revisan los conceptos de robustez, modularidad y capacidad evolutiva en el ámbito biológico, y se discute cómo podrían ser relevantes para la evolución de la arquitectura de Internet, así como para el diseño de arquitecturas futuras. También se identifican dos conceptos importantes en la evolución: los núcleos evolutivos y el equilibrio puntuado, que pueden explicar por qué los protocolos fundamentales en la arquitectura de Internet aparecen como osificados, al ser obligados a evolucionar a un ritmo mucho menor de manera que formen una base estable para todos los otros protocolos y mecanismos que dependen de ellos. Por último, se comenta otro objetivo de diseño, la optimización, que a menudo es de vital importancia en los diseños de ingeniería, pero que no parece de forma abundante en la biología.

\section{SELECCIÓN NATURAL Y GENÉTICA A LA DERIVA}

Las analogías y las metáforas pueden ser engañosas. Se podría argumentar que la evolución de los organismos vivos tiene muy poco en común con la evolución de un artefacto tecnológico, tal como Internet. Este argumento, sin embargo, pierde el sentido de que el proceso evolutivo es independiente del sustrato subyacente, y que es igualmente aplicable a los sistemas biológicos, naturales, tecnológicos o computacionales, siempre y cuando se mantengan unos principios fundamentales. Herbert Simon [2] fue uno de los primeros en argumentar convincentemente que el diseño de sistemas artificiales podría, y a menudo lo es, estar sujeto a los mismos principios que los sistemas naturales y biológicos.

El proceso evolutivo, en su forma más general, requiere los siguientes componentes: a) una población -una especie- de elementos -personas- con cada elemento especificado por genotipo; b) un mecanismo a través del cual se transfiere el genotipo de los individuos existentes, sujetos a variación -varios tipos de mutaciones o recombinación genética-, a nuevos individuos que pueden reemplazar a los viejos; c) un mecanismo -selección natural- que le de una ventaja reproductiva a los genotipos que mejor lo realicen, es decir, que tienen un fenotipo con mayor aptitud en el medio ambiente dado.

¿Cuáles son esos tres componentes para el caso de Internet? En primer lugar, Internet, considerada como una especie, consiste de millones de redes de computadores independientes pero interconectados entre sí, comenzando con las redes domésticas hasta los diferentes proveedores mundiales -estas redes son los individuos. Cada red se caracteriza por su genotipo: los protocolos y sistemas operativos que utiliza, las tecnologías utilizadas, las aplicaciones que los usuarios ejecutan, y así sucesivamente. En segundo lugar, constantemente las redes existentes son reemplazadas por las nuevas redes, con el genotipo de esta última fuertemente influenciado por el genotipo de sus predecesores. Por último, dependiendo del entorno más amplio -tecnologías y aplicaciones disponibles, costos económicos, pilas de protocolos existentes, etc.-, un genotipo de red dado puede dar lugar a un genotipo de mayor aptitud que otro; por ejemplo, la aptitud del actual protocolo X.25 sería mucho menor que la del IPv4 -esto no era necesariamente cierto en los años 80.

Los biólogos reconocen cuatro fuerzas evolutivas que pueden cambiar la frecuencia de las variantes genéticas en una población: 1) La mutación proporciona la materia prima, en forma de variación genética, para que se produzca la evolución; sin variación genética entre los individuos no puede haber 
cambio evolutivo. 2) La migración es el movimiento de los individuos -y sus genes- desde un lugar a otro, también llamado flujo de genes. 3) La selección Natural, que Darwin reconoció como la principal fuerza motora de la evolución, es un proceso determinístico, mediante el cual los rasgos que incrementan la supervivencia 0 el rendimiento reproductivo de los individuos -aptitud-, incrementa en frecuencia con el tiempo. 4) La genética a la deriva es un proceso estocástico, mediante el cual las variantes genéticas en una población cambian en frecuencia gracias al muestreo aleatorio y al azar. Considerando que la Selección Natural aumentará la frecuencia de las variantes genéticas aventajadas, la genética a la deriva puede producir variantes aventajadas, neutrales o perjudiciales para distribuirse mejor.

En la evolución, existe gran debate acerca de los roles relativos de la Selección Natural frente a la genética a la deriva. Lo que está claro es que el tamaño de una población dada determina la eficiencia con la que la selección natural puede actuar -los efectos aleatorios de la genética a la deriva son más fuertes en poblaciones pequeñas. Esto es similar a los efectos de muestreo de lanzar una moneda 10 veces ó 10.000 veces, por esto el rol de la casualidad será más dramático en una pequeña muestra.

El tema de la Selección Natural frente a la genética a la deriva puede ser muy relevante para la evolución de Internet. En los primeros días de Internet, cuando su población era de sólo un puñado de redes, era fácil para la arquitectura ir "a la deriva" de una forma a otra, simplemente debido al azar. Por ejemplo, la adopción de los primeros campos de encabezado TCP, debido a que rara vez se utilizaba el puntero URGENTE, puede explicarse como el resultado de "la deriva" -dado que su uso práctico fue y sigue siendo muy bajo. Hoy en día, Internet se compone de millones de redes y existe una enorme presión para adoptar cambios, y se hacen sólo si tendrán un impacto positivo sobre el estado de la red, es decir, la selección fuerte domina sobre "a la deriva" en la Internet actual. No debe sorprender que algunas propuestas recientes de arquitectura -como el despliegue de IP multicast O QoS- no hayan sido adoptadas actualmente, ya que no proporcionan un incremento al estado de la red(es).

\section{ROBUSTEZ}

La robustez es uno de los principios de diseño de mayor prevalencia en biología. Normalmente se define como la invariabilidad de ciertas características fenotípicas en la presencia de amplias perturbaciones internas o externas. Las variaciones internas son causadas por variabilidad genética, tales como mutaciones 0 variaciones en los parámetros bioquímicos de los mecanismos celulares, y las externas son causadas por las fluctuaciones en las condiciones ambientales -por ejemplo, la temperatura o la concentración de nutrientes. La robustez se define siempre en términos de características fenotípicas específicas -no es un atributo genérico de un sistema. Además, se define sobre una serie de perturbaciones -ningún diseño sería capaz de tolerar cualquier perturbación.

La robustez a menudo se describe como estabilidad, homeóstasis, o canalización, pero estas propiedades sólo son casos especiales de robustez. Por ejemplo, un proceso de desarrollo debe ser robusto a pesar de que su naturaleza dinámica significa que no puede ser estable. Además, la robustez requiere la invariancia de la función, no la invariancia de la estructura. Un organismo robusto puede ser significativamente plástico o variable, modificando su estructura interna para hacer frente a las perturbaciones dadas.

Algo importante que se puede aprender de la biología es que a pesar de que hay muchas maneras de proporcionar una funcionalidad determinada, muy pocas son realmente robustas para una amplia gama de perturbaciones. Esto se ha demostrado, por ejemplo, en el caso de la quimiotaxis bacteriana [3]. Los biólogos utilizan la robustez como criterio para seleccionar entre los diferentes modelos que puedan explicar el mismo efecto biológico: si un modelo conduce a la robustez con respecto a algunos parámetros clave, mientras que otro modelo requiere un fino ajuste en esos parámetros, el primero puede ser más plausible en determinadas circunstancias.

¿Por qué esperar que la robustez sea significativa para un sistema tecnológico como el Internet? Si el sistema funciona en un entorno estático, y siempre se diseñan sus componentes internos, la robustez sería una propiedad innecesaria y costosa. Sin embargo, los sistemas biológicos operan y evolucionan en ambientes impredecibles con componentes extremadamente desordenados. ¿Internet es diferente en ese aspecto? Un artefacto con el tamaño de Internet, con control heterogéneo y distribuido también se enfrenta a cambios importantes y frecuentes, tanto en sus condiciones ambientales -por ejemplo, mezcla de aplicaciones y ataques malintencionados- y en sus elementos internos -por ejemplo, routers, enlaces y protocolos. Posiblemente, una de las principales razones detrás del éxito de Internet es la solidez de la arquitectura TCP/IP con respecto a los cambios topológicos y las condiciones de sobrecarga.

El vínculo entre la robustez y la capacidad evolutiva es crítico. En principio, puede parecer que estas dos propiedades se contradicen entre sí: si un sistema puede mantener su función en todos los cambios del entorno, entonces no necesita evolucionar; y si un sistema es capaz de evolucionar cuando el entorno cambia, ¿para qué necesita ser robusto? Realmente no existe contradicción. La evolución se lleva a cabo en fases de tiempo largas y requiere de muchas generaciones, así como la aparición y/o lentitud de la variación genética. La robustez es importante para la viabilidad bajo entornos desfavorables, mientras que la evolución le permite a un sistema adatarse si/cuando las condiciones mejoran. 
¿Cómo logran ser robustos los sistemas biológicos? Parece que despliegan básicamente tres mecanismos. Uno es el uso extensivo de la modularidad y la jerarquía de diseño -se trata en sí mismo de un importante principio de diseño -se describe en la siguiente sección. Los otros dos mecanismos son el control de retroalimentación y la degeneración -se detallan a continuación.

\subsection{Control de retroalimentación}

La idea básica del control de la retroalimentación es utilizar la salida de un mecanismo para determinar la entrada correspondiente a dicho mecanismo. En el caso de la retroalimentación negativa, la entrada del mecanismo es la diferencia entre la salida observada y la deseada, un nivel de referencia que puede variar con el tiempo. Un sistema que despliega control de retroalimentación negativa puede lograr la robustez para las perturbaciones externas o internas si la escala de tiempo en los que la entrada varía es mayor que la escala de tiempo del controlador de la retroalimentación. Un tipo particular de retroalimentación negativa -referida como retroalimentación integral- integra el error entre la salida y la entrada de referencia a través del tiempo, y los intentos por minimizarla. El control integral de retroalimentación negativa se utiliza, por ejemplo, en la quimiotaxis bacteriana.

Los sistemas biológicos también utilizan la retroalimentación positiva. La cual puede forzar un sistema a uno de varios estados estables y a permanecer en ese estado incluso en presencia de desorden significativo en las entradas. Tales circuitos de retroalimentación positiva son comunes en los temas de redes, es decir, frecuentemente ocurren en subredes de Gene Regulatory Networks (GRNs) que se utilizan en desarrollo [4]. Por ejemplo, la diferenciación celular es un proceso que debe dar lugar a un tipo diferenciado y estable para cada celda, aunque sí existen son variaciones importantes en los parámetros cinéticos internos de la GRN correspondiente. Un ejemplo bien estudiado de robustez biológica es la llamada red de "polaridad de los segmentos" que determina la segmentación del cuerpo de la mosca de la fruta -Drosophila- [5]. La GRNs correspondiente se basa en varios circuitos de retroalimentación anidada, algunos de ellos positivos, y sus resultados en la segmentación de cuerpo robusto, incluso si los parámetros cinéticos subyacentes abarcan varias órdenes de magnitud.

El uso de control de retroalimentación, especialmente la retroalimentación negativa, es común en ciertos protocolos de redes informáticas -por ejemplo, en el control de congestión TCP y en diversos mecanismos de AQM-, pero menos comprendido en otros - por ejemplo, en la robustez de la ingeniería de tráfico en línea o en los circuitos de retroalimentación anidada que involucran tanto el transporte como las capas de la aplicación. Además, el uso de retroalimentación positiva para mejorar la robustez no se ha explorado lo suficiente. Por el contrario, a menudo se evita en la investigación en redes debido a su inestabilidad potencial.

\subsection{Redundancia y degeneración}

En los sistemas biológicos, la robustez también se proporciona a través de redundancia extensiva a todos los niveles, desde la codificación del código genético dentro de una célula hasta cómo se mueven o comunican los animales. Lo que es más interesante, sin embargo, es que la redundancia en biología no resulta simplemente desde múltiples copias de una determinada estructura o mecanismo, como se hace a menudo en ingeniería. En cambio, los sistemas biológicos logran la redundancia vía diferentes mecanismos estructurales que realizan la misma función -o funciones altamente superpuestas- y que operan de forma independiente y simultánea. Para distinguir los dos enfoques se adopta la terminología de Edelman [6], quien define redundancia como la replicación justa de la misma estructura, mientras que la degeneración significa que una función dada es proporcionada por diferentes estructuras y mecanismos.

En términos de robustez, la degeneración es más efectiva que la redundancia, a pesar de que probablemente en la ingeniería pueda verse como más costosas y complejas. Si una estructura o mecanismo que se basa en la redundancia, en determinadas condiciones, todas las réplicas de esa estructura podrán fallar simultáneamente cuando esas condiciones se encuentran. Dos ejemplos importantes de la degeneración biológica son la existencia de múltiples vías metabólicas paralelas para la misma función, o la activación de múltiples respuestas inmunes -por ejemplo, poblaciones de anticuerpos diferentes- en presencia de un patógeno.

La conexión entre la degeneración y la capacidad evolutiva es intrigante. En primer lugar, la degeneración puede incrementar la capacidad evolutiva. Este es el caso, por ejemplo, cuando los genes se duplican en los genomas y entonces acumulan mutaciones independientes. En segundo lugar, la degeneración puede resultar naturalmente desde el proceso evolutivo. Si una función es importante para la viabilidad, varios mecanismos pueden evolucionar de forma independiente para ofrecerla. A pesar de que la redundancia se utiliza comúnmente en las redes informáticas, especialmente para proteger los vínculos físicos o rutas de red de los errores, el uso de la degeneración, sus diferencias con la redundancia, y las consecuencias evolutivas de la degeneración para una arquitectura de red, en gran parte son inexplorados.

\section{MODULARIDAD}

La modularidad es una importante herramienta para el diseño de sistemas complejos. Cada módulo representa una parte relativamente autónoma del sistema total que es responsable por cierta función u objetivo. Los diferentes módulos se conectan entre sí para crear más funciones y sistemas complejos. 
Topológicamente hablando, si se piensa en un diseño como una red, la densidad de los bordes intra-módulos es mucho mayor que la densidad de los bordes intermódulos. Un concepto estrechamente relacionado es el de diseño jerárquico. Dos o más módulos se combinan para formar módulos más complejos en un nivel de arquitectura superior.

La modularidad también es abundante en biología [7]. Se ha observado en las GRN, redes metabólicas, redes de interacciones proteínicas, y en las redes de especies en ecosistemas. En el caso de GRNs, por ejemplo, se descubrió recientemente que las complejas se pueden representar como "circuitos biológicos" jerárquicos, conocidos como redes adorno [4]. Una red de adorno es un sub-grafo que aparece en una red real con frecuencia mucho más alta que en una red aleatoria de la distribución del mismo grado. Redes de adorno importantes, como Feedforward loops, BiFan o Dense-Overlapping Regulon, consisten de un pequeño número de genes interactivos que cumplen funciones específicas -como la supresión del ruido 0 la generación de pulso. La evolución, sorprendentemente, parece haber seleccionado pocos adornos para sus funciones en la construcción de sistemas más complejos.

La modularidad está estrechamente relacionada con la robustez. La modularidad particiona un diseño complejo en componentes más o menos independientes, una propiedad conocida como cuasidescomposición. Ruido, perturbaciones o fallos dentro de un módulo probablemente no afecten a otro módulo. Las variaciones de estado internas dentro de un módulo serían irrelevantes para otro módulo, a menos que afecten relativamente pocos vínculos entre los dos módulos.

Trabajos recientes de modelado en biología -en su mayoría utilizando modelos computacionales de procesos evolutivos simples- han proporcionado datos importantes acerca de la relación entre la modularidad y la capacidad evolutiva. En primer lugar, parece que la modularidad mejora la capacidad evolutiva: los diseños modulares pueden evolucionar más rápidamente y pueden dar lugar a diseños más complejos que los "monolíticos" [8]. A menudo, los diseños modulares evolucionan simplemente por reorganización de la conectividad inter-módulos, no por el diseño interno de los módulos individuales. En el desarrollo biológico, el desarrollo de GRNs de menor "cableado" puede causar grandes variaciones fenotípicas, e incluso puede explicar en algunos casos la evolución de nuevas especies [9].

En segundo lugar y de acuerdo con Modularly Varying Goals (MVGs) [8], la evolución de la modularidad se produce cuando el entorno cambia con el tiempo. La evolución con un objetivo constante, o en un entorno constante, conduce a optimización, pero no a diseños modulares. La razón es que, en un entorno constante, el proceso evolutivo gradualmente remueve la funcionalidad redundante, que puede ser de alguna manera provista por otros módulos, incrementando la densidad de los vínculos inter-módulos. Se trata de vínculos adicionales que gradualmente rompen la modularidad. La situación es muy diferente cuando el entorno cambia con el tiempo, y especialmente cuando ese entorno presenta Modularly Varying Goals. Con MVGs, los objetivos en cualquier punto del tiempo se pueden descomponer en objetivos simples y subfunciones; la jerarquía exacta de estos cambios de objetivos cambia con el tiempo, pero los bloques de construcción elementales subyacentes siguen siendo los mismos. Entonces, el proceso evolutivo selecciona diseños modulares en los que cada módulo subyacente implementa un objetivo elemental, mientras que la variación en el tiempo de los objetivos más complejos se lleva a cabo cableando los módulos subyacentes existentes. Las simulaciones de [8] han puesto de manifiesto que la evolución en MVGs progresa mucho más rápido que la evolución con un objetivo constante o al azar con diferentes objetivos.

Las interacciones previas entre la modularidad, la robustez y la capacidad evolutiva son relevantes tanto para la evolución de la arquitectura existente de Internet como para la creación de nuevas, pero evolucionables, arquitecturas de redes. En primer lugar, se debe aceptar que la modularidad y la jerarquía son herramientas necesarias para hacer frente a la complejidad. Recientes propuestas para arquitecturas de red sin capas, o para optimizaciones de capa en cruz que reducen la modularidad del sistema en general, probablemente se mueve en la dirección equivocada. Optimizar una métrica particular, tales como el consumo de energía o la capacidad de la red, puede ser mucho menos importante que la capacidad evolutiva a la robustez a largo plazo de la arquitectura propuesta. En segundo lugar, las arquitecturas de red que están diseñadas para hacer frente a un entorno que varía en el tiempo, en términos de las limitaciones, las tecnologías disponibles, los costos, los requisitos de las aplicaciones, etc., se espera que evolucionen más rápido cuando se reorganizan y utilizan como los módulos existentes, en comparación con las arquitecturas que se basan completamente en módulos y funciones nuevas cada vez que cambia el entorno.

\section{SEMILLAS EVOLUCIONARIAS}

Existe una creciente evidencia biológica de que ciertos genes, y en particular, ciertas Gene Regulatory Networks GRNs, han sufrido muy pocos cambios en por lo menos la mitad de la duración de la vida en este planeta. Por ejemplo, ciertos desarrollos de GRNs se establecieron en el cámbrico temprano -alrededor de 510 millones de años- y no han evolucionado significativamente desde entonces [9]. Estos GRNs se conocen como semillas, y ahora se sabe que son en gran parte responsables de los aspectos principales de los planos del cuerpo de los animales. La existencia de planos a nivel básico indica que pudieron haber sido formados, en gran medida, por la acción de estas semillas GRNs. Por ejemplo, el corazón de la mosca de la fruta y el corazón de un ser humano, a pesar de 
tener morfologías distintas, fomentan el uso del mismo núcleo GRN cardiaco.

¿Por qué las semillas GRNs resisten el cambio evolutivo? En palabras de Davidson \& Erwin [9], "el cambio de los mismos está prohibido bajo pena de una catástrofe en el desarrollo, tanto por su cableado recursivo interno como por su importante rol en el desarrollo de la jerarquía de la red". Las semillas evolutivas representan una base estable sobre la que la diversidad y la complejidad de los procesos de más alto nivel pueden evolucionar. Por otra parte, las semillas GRNs limitan los ejes principales del desarrollo y la morfología y así, inevitablemente, limitan el alcance de los planos del cuerpo que posteriormente puede evolucionar.

Las semillas evolutivas también existen, y pueden desempeñar un papel similar, en la evolución de los artefactos tecnológicos como la arquitectura de Internet. El IPv4, el protocolo central de Internet, fue el primer protocolo -junto con TCP-que se creó en la arquitectura de Internet y que ha evolucionado muy lentamente desde entonces. Aun cuando se redefinieron ciertos campos de su cabecera, estos cambios fueron siempre compatibles sin necesidad de ningún cambio en las redes existentes. Es posible que el IPv4 desempeñe el papel de una semilla evolutiva en la arquitectura de Internet, y se una junto a la amplia gama de protocolos y tecnologías para las capas física y de enlace de datos, con la gran diversidad de protocolos, aplicaciones y servicios para las capas superiores de la arquitectura.

IPv4 puede funcionar sobre cualquier tecnología de enlace, y presumiblemente cualquier aplicación puede funcionar a través de IP. Esto sería mucho más difícil, si fuera posible, si el IPv4 estuviera sujeto a cambios frecuentes, o si existieran varios protocolos en el mismo nivel con el IPv4 -ya que ello crea la necesidad de "traducción" entre ellos. Al mismo tiempo, el protocolo IPv4 limita el espacio de los posibles protocolos que pueden existir por debajo o por encima de él. Sería un interesante proyecto de investigación identificar de alguna manera los límites de los servicios que pueden proveerse a través del IPv4. Si una arquitectura futura de Internet tiene como objetivo sustituir al IPv4, su mejor apuesta puede ser el soporte de aplicaciones y servicios que están por fuera de los límites del IPv4, asumiendo por supuesto que hubiese interés real por tales servicios.

\section{EQUILIBRIO PUNTUADO}

Hoy en día, la mayoría de los biólogos coinciden en que la evolución no procede a un ritmo más o menos constante -conocido como gradualismo-, sino que se alterna entre largos períodos de estancamiento y períodos relativamente cortos de intenso cambio en las especies existentes y en los mecanismos de desarrollo subyacentes que producen esas especies [10]. Este patrón evolutivo es conocido como equilibrio puntuado. Por ejemplo, la "explosión cambriana" se refiere a un período de tiempo relativamente corto, alrededor de
530 millones de años, en el que el ritmo de evolución aumentó en un factor de diez conduciendo la principal diversificación de las especies. Recientemente, Jain \& Krishna [11] propusieron un modelo evolutivo computacional que muestra cómo el proceso evolutivo puede proceder con equilibrio puntuado bajo supuestos muy generales, sin considerar necesariamente un sustrato biológico. Su modelo considera una red co-evolucionaria de interacciones, tanto positivas como negativas, entre un número de especies. El concepto de equilibrio puntuado también ha sido aplicado en el contexto de la teoría social y en el estudio de la difusión tecnológica.

Se plantea entonces la intrigante hipótesis de que la arquitectura de Internet también está sujeta al equilibrio puntuado. Un estudio empírico de esta hipótesis requeriría una medición del ritmo con el que la arquitectura de Internet adopta los nuevos protocolos o modificaciones significativas a sus protocolos -una difícil pero no imposible tarea.

¿Qué pasa si la hipótesis del equilibrio puntuado es cierta? En primer lugar, no debemos esperar que la arquitectura de Internet evolucione a un ritmo más o menos constante. Es "natural" para un artefacto tecnológico como Internet pasar por largos períodos de inmovilización, durante el cual su arquitectura parece estar osificada. En segundo lugar, el modelo de JainKrishna muestra que aunque la mayoría de los estímulos externos no inician cambios evolutivos significativos, pueden existir raros y menores eventos externos que pueden disparar cambios significativos en la organización interna de un ecosistema. Sin embargo, en el modelo de Jain-Krishna estos no son hechos aleatorios, ya que afectan a la así llamada dominante Auto-Catalytic Set (ACS) en el ecosistema, ya sea dañándolo o cambiándolo a otro conjunto de especies. Ésta puede ser una consideración importante para los investigadores que trabajan en diseñar la futura arquitectura de Internet. Una nueva arquitectura podría reemplazar o afectar significativamente la existente sólo si ofrece una ventaja importante en esas funciones básicas que desempeñan el papel de la dominante ACS actual; de lo contrario no podrá tener impacto o, en el mejor de los escenarios, sería absorbida por la arquitectura existente de Internet.

\section{IDONEIDAD}

En la literatura de ingeniería, incluyendo el estudio de las redes informáticas, optimizar en términos de rendimiento o costos suele ser el principal objetivo -a menudo, el único objetivo. En muy pocos casos -sobre todo en el diseño de la topología-, el problema de la optimización incluye algunas restricciones de fiabilidad para hacer frente a la vinculación limitada o a nodos de escenarios de fracaso. Rara vez se considera el problema de robustez más general, cuyo objetivo es mantener el buen funcionamiento del sistema ante variaciones internas o externas. Otros objetivos, como la capacidad evolutiva o la modularidad son aún menos frecuentemente mencionados. 
Por otro lado, los sistemas biológicos parecen ser "diseñados" principalmente para robustez, modularidad y capacidad evolutiva. Si pensamos en la robustez y la modularidad como mecanismos que facilitan la evolución, es justo decir que el objetivo principal de los sistemas biológicos esta última. Por el contrario, los sistemas biológicos no parecen ser globalmente óptimos en términos de estructura o función [12]; un ingeniero usualmente puede diseñar algo para superar a un organismo en una simple tarea. ¿Por qué se da eso?

Los sistemas modulares y sólidos típicamente no son óptimos topológicamente -es decir, en términos del número de sus componentes y conexiones internas-, ni dinámicamente -es decir, en términos de la dinámica de su respuesta. En primer lugar, la topología de los sistemas modulares incluye, como se señaló anteriormente, a elementos funcionales redundantes $\mathrm{e}$ interconexiones. La razón es que cada módulo tiene que realizar con independencia sus funciones, incluso si algunos elementos de esa función están también disponibles en otros módulos. Además, la topología de los sistemas sólidos es altamente redundante, ya sea porque algunos elementos funcionales se replican o porque hay múltiples y diferentes elementos que implementan la misma función -degeneración. Esta redundancia topológica incrementa el costo estructural y operacional del sistema resultante, por lo que no se considera óptima. En segundo lugar, en términos de la dinámica de respuesta, los sistemas modulares típicamente son más lentos y a menudo tienen que pasar por estados transitorios intermedios antes de llegar a un atractor -un punto que representa un estado de equilibrio, mientras que un ciclo límite supondría un comportamiento oscilatorio. Los sistemas sólidos también son más lentos debido a todas las etapas de procesamiento adicional, controles y ciclos de retroalimentación que requieren solidez.

En algunos casos, los sistemas biológicos parece ser la optimización de una cierta relación de costobeneficio, pero de una manera heurística que se asemeja a las técnicas de búsqueda estocástica. Un buen ejemplo es el circuito regulatorio genético que controla la producción de lactosa en la E-coli. En esa bacteria, existe una clara desventaja de costobeneficio: la célula crece más lento de lo que se produce la lactosa, mientras que por otro lado, descompone lentamente la lactosa que se utilizará como fuente de energía para ayudar a la célula a crecer más rápido. E-coli tiene un mecanismo de regulación que le permite a una población de células avanzar hacia el nivel óptimo de producción de la lactosa, es decir, el nivel que maximiza la tasa de crecimiento de la célula. En otras palabras, una población de células E. coli es absorbida rápidamente por las células que optimizan su nivel de producción de lactosa.

¿Cómo implica esto las arquitecturas de red y en particular la de Internet? En primer lugar, si se acepta que el diseño de la arquitectura de una red no es estático, sino que evoluciona constantemente por la presión de los requisitos de nuevas aplicaciones, las restricciones económicas y las tecnologías disponibles, se tiene que aceptar que la arquitectura debe ser diseñada principalmente para evolucionar, no para optimizar. Las herramientas que promuevan la capacidad evolutiva, tales como modularidad, jerarquía, robustez y degeneración pueden ser tan o más importantes que el rendimiento o el costo. En segundo lugar, los componentes arquitectónicos que se deben optimizar, en su caso, son los que proporcionan una ventaja evolutiva a la arquitectura. Se debe evitar las optimizaciones que ponen en peligro la capacidad evolutiva de la arquitectura, incluso si se traducen en beneficios a corto plazo. En tercer lugar, ninguna optimización debe ser adaptable a la naturaleza, tratando de optimizar heurísticamente importantes concesiones, incluso en entornos desconocidos y variables en el tiempo. Optimizaciones que suponen el completo conocimiento del entorno en el que opera la arquitectura, o que requieren que el entorno sea constante, pueden ser pasos en la dirección equivocada.

\section{CONCLUSIONES}

En este trabajo se han revisado algunos conceptos importantes en la evolución biológica, especialmente relacionados con capacidad evolutiva, y se discutió cómo esos conceptos pueden ser relevantes para la evolución de las arquitecturas de Internet. Un próximo paso importante, actualmente en estudio, es aplicar esos conceptos en un marco cuantitativo, a través de enfoques experimentales o teóricos, en problemas específicos de diseño de redes. Por ejemplo, el uso de la degeneración, en vez de la redundancia; el impacto de los objetivos de diversas modularidades en la capacidad evolutiva de una arquitectura de red, o el equilibrio entre la optimización y la capacidad evolutiva, son líneas de investigación que parecen fértiles y deberían continuarse. Se espera que en el futuro estas líneas de investigación den lugar a más trabajos interdisciplinares entre científicos de redes y biólogos.

\section{REFERENCIAS}

[1] M. Kirschner \& J. Gerhart. "Evolvability". PNAS, Vol. 95, No. 15, pp. 8420-8427, July 1998.

[2] H. A. Simon. "The Sciences of the Artificial". USA: MIT Press, 215 p. 1996.

[3] U. Alon, M. G. Surette, N. Barkai \& S. Leibler. "Robustness in Bacterial Chemotaxis". Nature, Vol. 397, pp. 168-171, January 1999.

[4] R. Milo, S. Itzkovitz, N. Kashtan, R. Levitt, S. Shen-Orr, I. Ayzenshtat, M. Sheffer \& U. Alon. "Superfamilies of Evolved and Designed Networks". Science, Vol. 303, No. 5663, pp. 1538-1542, March 2004.

[5] G. von Dassow, E. Meir, E. M. Munro \& G. M. Odell. "The Segment Polarity Network is a Robust Developmental Module". Nature, Vol. 406, pp. 188-192, July 2000.

[6] G. M. Edelman \& J. A. Gally. "Degeneracy and Complexity in Biological Systems". PNAS, Vol. 98, No. 24, pp. 13763-13768, November 2001. 
[7] L. Hartwell, J. Hopfield, S. Leibler \& A. Murray. "From Molecular to Modular Cell Biology". Nature, Vol. 402, pp. C47-C52, December 1999.

[8] N. Kashtan, E. Noor \& U. Alon. "Varying Environments can Speed Up Evolution". PNAS, Vol. 104, No. 34, pp. 13711-13716, August 2007.

[9] E. H. Davidson \& D. H. Erwin. "Gene Regulatory Networks and the Evolution of Animal Body Plans". Science, Vol. 311, No. 5762, pp. 796-800, February 2006.
[10] S. J. Gould. "The Structure of Evolutionary Theory". USA: Harvard University Press, 1464 p. 2002.

[11] S. Jain \& S. Krishna. "Large Extinctions in an Evolutionary Model: The Role of Innovation and Keystone Species". PNAS, Vol. 99, No. 4, pp. 20552060, February 2002.

[12] G. A. Parker \& J. Maynard Smith. "Optimality Theory in Evolutionary Biology". Nature, Vol. 348, pp. 27-33, November 1990. 